\title{
Avaliação da qualidade das máscaras comercializadas no Brasil em tempos de pandemia da COVID-19 quanto à presença de prata e de nanopartículas de prata
}

\section{Quality evaluation of masks marketed in Brazil during the COVID-19 pandemic for the presence of silver and silver nanoparticles}

\author{
Cristiane Barata-Silva',* iD \\ Santos Alves Vicentini Neto' iD \\ Carolina Duque Magalhães' \\ Silvana Couto Jacob' iD \\ Josino Costa Moreira" (iD
}

Lisia Maria Gobbo dos Santos'

I Instituto Nacional de Controle de Qualidade em Saúde (INCQS), Fundação Oswaldo Cruz (Fiocruz), Rio de Janeiro, RJ, Brasil

" Centro de Estudos da Saúde do Trabalhador e Ecologia Humana (CESTEH), Escola Nacional de Saúde Pública Sergio Arouca (ENSP), Fundação Oswaldo Cruz (Fiocruz), Rio de Janeiro, RJ, Brasil

\footnotetext{
* E-mail: cristianebarata@hotmail.com
}

Recebido: 26 ago 2020

Aprovado: 04 nov 2020

\section{RESUMO}

Introdução: A pandemia COVID-19 causada pelo novo coronavírus é uma doença sem tratamento específico que se tornou um dos grandes desafios do século atual. Uma das alternativas para minimizar a taxa de transmissão direta do vírus é o uso das máscaras faciais. Atualmente, há no mercado uma diversidade de máscaras, tanto na composição têxtil, quanto na presença ou não do elemento prata (Ag), sob a forma de íon ou nanopartículas de prata (AgNP), que possui atividade biocida. Objetivo: Avaliar a presença da Ag total e de AgNP em máscaras produzidas para proteger a população da COVID-19 que estão sendo comercializadas no Brasil durante a pandemia. Método: $O$ desenho utilizado para o estudo foi observacional descritivo do tipo transversal com amostragem por conveniência. As amostras foram analisadas por ICP-MS no modo padrão e no modo single particle. Resultados: As concentrações de prata total nas amostras estudadas apresentaram uma variação de $14-72 \mu \mathrm{gg}^{-1}$. Foi observado que $50 \%$ das amostras avaliadas que declaram ter AgNP apresentaram uma distribuição de tamanho entre 17-57 nm. Ao serem submetidas aos ciclos de lavagem, verificou-se uma redução na concentração de Ag, a cada novo ciclo, o que levanta o questionamento quanto a sua real efetividade biocida ao longo do tempo. Conclusões: Os dados gerados fornecem o atual cenário da concentração de Ag nas máscaras e, assim, avaliar o potencial benefício ou risco do uso para a saúde humana e ambiental. Além disso, este conhecimento pode dar subsídios técnico-científicos para a fiscalização sanitária do controle de qualidade e a implementação de normas regulatórias nesse ramo de atuação.

PALAVRAS-CHAVE: COVID-19; Pandemia; Prata; Nanopartículas; ICP-MS

\section{ABSTRACT}

Introduction: The COVID-19 pandemic caused by the new coronavirus is a disease without specific treatment and has become one of the great challenges of the current century. One of the alternatives to minimize the direct transmission rate of the virus is the use of face masks. Currently, there is a diversity of masks on the market, both in the textile composition and in the presence or absence of the silver (Ag) element, in the form of ions or silver nanoparticles (AgNP), which have biocidal activity. Objective: To evaluate the presence of total Ag and AgNP in masks produced to protect the population from COVID-19 being sold in Brazil during the pandemic. Method: This is a cross-sectional descriptive observational study with convenience sampling. The samples were analyzed by ICP-MS in the standard mode and in the single particle mode. Results: The concentrations of total Ag in the studied samples varied from 14 to $72 \mu \mathrm{gg}^{-1}$. It was observed that $50 \%$ of the evaluated samples that claim to have AgNP had a size distribution between 17 and $57 \mathrm{~nm}$. When subjected to the washing cycles, there was a reduction in the concentration of $\mathrm{Ag}$, which raises the question as to its real biocidal effectiveness over time. Conclusions: The data generated can provide the current scenario of the concentration of silver in the masks and thus assess the potential benefit or risk of use for human and environmental health. In addition, this knowledge can provide technical-scientific support for the sanitary inspection of quality control and implementation of regulatory standards in this field of activity.

KEYWORDS: COVID-19; Pandemic; Silver; Nanoparticles; ICP-MS 


\section{INTRODUÇÃOO}

A COVID-19, doença infectocontagiosa causada pelo SARS-CoV-2, é uma doença sem tratamento específico que se tornou um dos grandes desafios do século $X X^{1}$. Por ser uma doença causada por um vírus de transmissão no trato respiratório, a COVID-19 se dissemina principalmente por gotículas, secreções respiratórias e contato direto com paciente infectado, sendo transmitida de humano para humano (transmissão direta) ${ }^{1}$. Em função dessas características, atualmente, a COVID-19 está presente em mais de 100 países e cinco continentes ${ }^{2}$, sendo, assim, classificada como uma pandemia.

Devido à falta de vacina e de tratamento específico, as medidas mais eficazes de evitar contaminação se baseiam em impedir a propagação do vírus de pessoa para pessoa através do isolamento ou distanciamento social. Além disso, os órgãos competentes na área da saúde recomendam o uso de máscaras que pode contribuir para diminuir a propagação dessa doença em larga escala, objetivando uma proteção coletiva ${ }^{3}$. A máscara funciona como uma barreira física, diminuindo a contaminação do vírus por meio de respingos de espirros ou tosses, além de evitar a contaminação mão-boca ${ }^{4}$.

Em função disso, a indústria têxtil está investindo em tecidos com atividade antimicrobiana, utilizando a prata (Ag) que é um elemento natural e com características físico-química, óptica e biológica únicas. Além disso, a Ag é bem conhecida pela sua forte toxicidade para uma vasta gama de microrganismos ${ }^{5}$. Atualmente a $\mathrm{Ag}$, vem sendo adicionada a vários produtos de consumo, incluindo roupas, geladeiras e máquinas de lavar para desodorizar ou higienizar, embalagens plásticas para armazenar alimentos e, também, na água potável para fins desinfetantes, sem nenhum dano à saúde ainda descrito ${ }^{6}$.

Entretanto, a forma como a Ag está presente nesses produtos é relevante. A forma iônica da Ag exibe atividade bactericida pela inibição de uma série de processos biológicos de bactérias, principalmente, Gram-negativas e vem sendo usada há muito tempo, sem apresentar atividade carcinogênica ou mutagênica nos seres vivos ${ }^{7}$. Porém, esse íon possui baixa estabilidade pois tende a reagir com ânions como $\mathrm{Cl}^{-}, \mathrm{HS}^{-} \mathrm{eSO}_{4}^{-}$em água formando precipitados que diminuem sua atividade biocida ${ }^{8,9}$.

Com o surgimento da nanotecnologia, uma ciência multidisciplinar e considerada inovadora, foi possível produzir substâncias em escalas nanométricas ${ }^{10,11,12} \mathrm{e}$, assim, ter seus efeitos potencializados, como, por exemplo: a utilização de nanopartícula de prata (AgNP), que possui atividade antimicrobiana comprovada. Sendo assim, as AgNP podem ser usadas em máscaras como barreira superficial, reduzindo o número de patógenos e a chance de contaminação e podendo combater o vírus SARS-CoV-2 ${ }^{8,9}$.

O objetivo deste estudo foi avaliar a presença da Ag total e de AgNP em máscaras produzidas para proteger a população do vírus SARS-CoV-2 que estão sendo comercializadas no Brasil durante o período da pandemia da COVID-19. Os dados gerados fornecerão aos órgãos competentes o atual cenário da concentração de prata nas máscaras comercializadas. Além disso, a validação da técnica poderá ser utilizada por outros laboratórios para controle desses produtos, desde que realizem uma validação intralaboratorial.

\section{MÉTODO}

Este estudo foi realizado no Setor de Elementos Inorgânicos do Departamento de Química do Instituo Nacional de Controle de Qualidade em Saúde (INCQS), unidade da Fundação Oswaldo Cruz (Fiocruz).

Os experimentos foram realizados em um espectrômetro de massas com plasma indutivamente acoplado operado no modo padrão e no modo single particle (sp-ICP-MS), modelo NexION 300D (Perkin Elmer, EUA). O espectrômetro de massa com plasma indutivamente acoplado (ICP-MS) estava equipado com um nebulizador concêntrico (Meinhard), câmara de nebulização ciclônica de vidro, cone, skimmer e hiper-skimmer de níquel. Gás argônio com pureza mínima de $99,996 \%$ foi fornecido pela White Martins (São Paulo, Brasil). Os parâmetros instrumentais e de aquisição de dados estão listados no Quadro 1.

\section{Padrões}

Suspensões monodispersas de nanopartículas esféricas de prata foram preparadas a partir de soluções padrões de $40 \mathrm{~nm}$ e $50 \mathrm{~nm}$ (Sigma Aldrich - San Luis, Missouri, EUA), contendo 7,2 x $10^{10} \mathrm{e}$ $7,4 \times 10^{9}$ partículas $/ \mathrm{mL}$, respectivamente.

As suspensões de nanopartículas foram sonificadas por $1 \mathrm{~min}$ com uma frequência de $25 \mathrm{kHz}$, após diluições sucessivas, e preparadas em água deionizada (Milli-Q Advantage, Molsheim, França). Após a diluição e antes de cada análise, as suspensões foram sonificadas por 1 min com uma frequência de $25 \mathrm{kHz}$ e homogeneizadas em vortex por $1 \mathrm{~min}$.

Soluções de Ag dissolvida para confecção da curva de calibração (1, $5,10,15,20 \mu \mathrm{gL}^{-1}$ ) foram preparadas por meio de diluições sucessivas em água deionizada a partir da solução-padrão estoque de $1 \mathrm{mgL}^{-1}$

Quadro 1. Parâmetros instrumentais para análise por espectrometria de massa com plasma indutivamente acoplado (ICP-MS).

\begin{tabular}{|lcc|}
\hline Parâmetros instrumentais & & \\
\hline Radio Frequência (RF) Power & $1.400 \mathrm{~W}$ & \\
Fluxo do argônio & & \\
Plasma & $18 \mathrm{Lmin}^{-1}$ & \\
Auxiliar & $1,2 \mathrm{Lmin}^{-1}$ & \\
Nebulizador & $1,0 \mathrm{Lmin}^{-1}$ & \\
Fluxo da amostra & $0,2 \mathrm{mLmin}^{-1}$ & \\
Parâmetros de aquisição & & \\
Unidade de mediada & padrão & Single particle \\
Varredura & 20 & 1 \\
Dwell time & $50 \mathrm{~ms}^{-1}$ & $50 \mu \mathrm{s}$ \\
Replicatas & 1 & 200.000 \\
Tempo de integração & $1 \mathrm{~s}$ & $100 \mathrm{~s}$ \\
\hline
\end{tabular}

Fonte: Elaborado pelos autores, 2020. 
(Sigma Adrich San Luis, Missouri, EUA). Como padrão interno foi utilizada solução de ródio (Rh) de $1.000 \mathrm{mgL}^{-1}$ (Merck, Alemanha).

\section{Amostras}

O desenho utilizado para o estudo é observacional descritivo do tipo transversal, com a descrição e análise das máscaras contendo Ag sob a forma de íons ou de AgNP que estão sendo atualmente comercializadas no Brasil, com fabricação caseira ou industrial. 0 tipo de amostragem adotado foi por conveniência de forma aleatória, sendo que as amostras foram adquiridas no mercado nacional em diferentes estabelecimentos comerciais localizados no estado do Rio de Janeiro e através da internet.

No período de maio a julho de 2020 , foram selecionadas amostras de máscaras de proteção de diferentes valores de aquisição, composição têxtil. Foram analisadas dez amostras de máscaras, identificadas por letras, sendo que seis máscaras declararam ter em sua composição Ag (A-F) e ação anti-COVID-19 conforme a ISO $18184: 2019^{13}$ e quatro (G-J) não declararam tal função em seu material de divulgação no mercado nacional, utilizadas como controle no estudo.

\section{Preparação das amostras}

Cada amostra (máscara) foi dividida em quadrantes de $3 \mathrm{~cm}^{2}$ (equivalente $0,1 \mathrm{~g}$ ) para que os pedaços analisados tivessem a mesma área. Esses pedaços foram selecionados de forma que os resultados obtidos representassem a concentração de $\mathrm{Ag}$ presente em toda a área da amostra. Para tal, os quadrantes analisados foram os presentes na extensão das duas diagonais dos tecidos, buscando sempre compreender as extremidades e a região central da máscara.

\section{Prata total}

As máscaras foram submetidas a uma digestão ácida por micro-ondas modelo Speed Wave (Berghof, Alemanha) por $45 \mathrm{~min}^{14,15}$. Neste procedimento, cerca de $0,3 \mathrm{~g}$ (cerca de três quadrantes de $3 \mathrm{~cm}^{2}$ ) da amostra de tecido foi pesado em hexaplicata e acondicionada em tubos de plástico tipo Teflon, no qual foram adicionados $3 \mathrm{~mL}$ de água deionizada (Millipore, Brasil) e $5 \mathrm{~mL}$ de ácido nítrico suprapuro $65 \%(\mathrm{p} / \mathrm{v})$ (Merck, Alemanha). Após o tempo de digestão e resfriamento dos tubos, as soluções resultantes do processo de digestão das amostras foram transferidas para frascos volumétricos de $15 \mathrm{~mL}$, adicionadas de padrão interno Rh na concentração final de $10 \mu \mathrm{gL}^{-1} \mathrm{e}$ o volume final preenchido com água ultrapura.

\section{Nanopartículas de prata}

Cerca de $0,3 \mathrm{~g}$ (cerca de três quadrantes de $3 \mathrm{~cm}^{2}$ ) da amostra foi pesado em triplicata e cortada em fragmentos $\leq 5 \mathrm{~mm}$, para aumentar a superfície de contato da amostra com a água, e acondicionada em tubos tipo Falcon, no qual foram adicionados $15 \mathrm{~mL}$ de água e, em seguida, as amostras foram submetidas a ultrassom por $2 \mathrm{~h}$, centrifugação em velocidade de $4.500 \mathrm{rcf}$ por 30 min e filtração em membrana de porosidade de $0,22 \mu \mathrm{m}$.
A etapa de filtração foi usada para remover matéria orgânica indesejada. Nesta etapa podem ocorrer perdas de partículas devido ao tamanho ou por possível interação com o material do filtro, mas estas não foram consideradas neste estudo. As amostras foram preparadas em triplicata.

Perdas da Ag no processo de lavagem

As amostras tendo Ag declarada na sua composição foram submetidas ao processo de lavagem e secagem seguindo as orientações da Agência Nacional de Vigilância Sanitária (Anvisa) ${ }^{16}$ e dos fabricantes. Foram feitos 30 ciclos de lavagem para avaliar se ocorre perda da concentração total da Ag presente nos tecidos. A cada cinco ciclos de lavagem e secagem, uma quantidade de $0,1 \mathrm{~g}$ de cada máscara (equivalente a $3 \mathrm{~cm}^{2}$ ) era coletada e a Ag total determinada. As análises foram realizadas em duplicata.

\section{Validação da metodologia}

O método analítico foi validado intralaboratorialmente de acordo com os parâmetros descritos no documento de orientação sobre Validação de Métodos Analíticos do Instituto Nacional de Metrologia, Qualidade e Tecnologia (Inmetro) (DOQ-CGCRE-008) ${ }^{17}$ e da Norma ABNT NBR ISO/IEC 17025:201718. A faixa de trabalho estabelecida foi 1-20 $\mu \mathrm{gL}^{-1}$. 0 limite de detecção para Ag total $\left(\operatorname{LOD}_{\mathrm{T}}\right)$ no modo padrão foi obtido pela leitura de dez replicatas independentes da solução-branco e calculado, com um grau de confiança de $95 \%{ }^{17,19}$.

A identificação e quantificação das AgNP pelo sp-ICP-MS depende de dois fatores: (i) o tamanho da nanopartícula, que deve ser grande o suficiente para gerar um número de íons detectável pelo espectrômetro, e (ii) a concentração numérica de nanopartículas, que deve ser alta o suficiente para permitir contagem de um número mínimo de eventos. Em função disso, dois limites de detecção são calculados: limite de detecção de tamanho ( LOD $_{d}$, diâmetro para nanopartículas sólidas) e limite de detecção da concentração do número de nanopartículas $\left(\operatorname{LOD}_{\mathrm{NP}}\right)$. Os $\operatorname{LOD}_{\mathrm{d}}$ e $L_{\mathrm{NO}}$ no modo sp-ICP-MS foram obtidos experimentalmente através da média da leitura de dez soluções independentes do branco com tempo de permanência de $50 \mu$ s (dwell time), fluxo da amostra $0,2 \mathrm{mLmin}^{-1}$ e eficiência de transporte (TE) de 8,87\%17,19,20.

Para avaliação do efeito matriz, uma curva analítica preparada em solvente (água) foi comparada com uma curva preparada na presença da amostra ${ }^{21}$. 0 parâmetro da recuperação foi avaliado no modo padrão e single particle usando três vezes a quantidade de $0,3 \mathrm{~g}$ da mesma máscara. Cada pedaço foi fortificado com uma solução final de prata de $30 \mu \mathrm{gL}^{-1} \mathrm{e}$, em seguida, submetido a digestão por micro-ondas. Para AgNP, os tecidos foram fortificados com uma solução contendo partículas de tamanho médio de $40 \mathrm{~nm}$ com $50.000 \pm 1.000$ partículas $/ \mathrm{mL}$ e estes ficaram em repouso por $24 \mathrm{~h}$ para absorção da solução pelo tecido. Após secura total do tecido foi realizado o preparo de amostra conforme descrito anteriormente. A repetibilidade, expressa em desvio-padrão relativo (SDR), foi avaliada usando as leituras de cinco repetições das amostras feitas no modo padrão e no modo single particle, sob as mesmas condições analíticas, menor espaço de tempo possível e mesmo analista ${ }^{17,21}$. 


\section{Análise estatística}

Uma análise estatística descritiva foi realizada no software Microsoft Excel 2010, compreendendo as médias aritméticas e desvio-padrão. Além disso, foi aplicado o teste $t$ de Student e o teste estatístico de análise de variância (Anova) para comparação das médias. A avaliação da linearidade da curva analítica foi avaliada com o auxílio da planilha intitulada "planilha para avaliação de premissas", elaborada por Bazilio et al. ${ }^{22}$.

\section{RESULTADOS E DISCUSSÃO}

Os resultados obtidos na validação da metodologia para determinação de Ag e AgNP no modo padrão e no modo single particle estão apresentados na Tabela 1.

O efeito matriz, a recuperação e a repetibilidade foram avaliados de acordo com Inmetro ${ }^{17}$. Para avaliar a recuperação e a precisão foram selecionadas três amostras de máscara, duas que declaravam ter prata na sua composição e uma que não declarava ter prata na sua composição. Os resultados referentes à recuperação e à precisão estão apresentados na Tabela 2.

As curvas analíticas foram consideradas homogêneas, com um nível de confiança de $95 \%$ e comparadas através do teste $\mathrm{t}$ de Student. Por esse teste foi possível concluir que as curvas se equivalem, isto é, a matriz não apresenta interferência significativa na quantificação da Ag nas amostras estudadas. Os resultados de avaliação de recuperação e repetibilidade estão adequados com recuperações obtidas dentro da faixa aceitável 60\%-115\% e a porcentagem do desvio-padrão relativo (\% RSD) não ultrapassou $21 \%$, segundo preconizado pelo documento orientativo do Inmetro ${ }^{17}$.

Segundo levantamento bibliográfico, o estudo da exatidão deve ser avaliado usando material de referência certificado (MRC), uma vez que fatores como a matriz, o tamanho, a densidade, a estequiometria e o tipo de nanopartícula influenciam a recuperação ${ }^{13}$. Não foi possível usar MRC uma vez que não foi encontrado disponível para aquisição tanto no mercado nacional quanto no internacional, contudo, os resultados obtidos demostram que o método apresenta uma boa exatidão.

Identificação e quantificação de Ag total e AgNP nas amostras

Cada amostra foi preparada conforme descrito anteriormente e, para cada replicata, foram realizadas cinco leituras independentes, sendo que os resultados foram utilizados para o cálculo da média e desvio-padrão, como mostrado na Tabela 3.

Ao comparar os resultados das amostras analisadas, observa-se que foi possível quantificar $\mathrm{Ag}$ em todas as amostras, mesmo nas amostras que não declararam ter Ag na sua composição. A variação observada foi na concentração, pois nas amostras que não declararam ter $\mathrm{Ag}$, a concentração foi menor que $2 \mu \mathrm{gg}^{-1}$, enquanto as amostras que declararam ter Ag na sua composição o valor encontrado foi no mínimo sete vezes maior, com exceção da amostra F, que declarou ação antimicrobiana, e a concentração da Ag estava próxima à das amostras que declararam não ter essa ação, entretanto essa ação pode ser causada por outras substâncias não avaliadas nesse estudo.

Das seis amostras que declararam ter ação antiviral nenhuma declarou a concentração de prata no tecido e somente duas amostras declararam ter sido comprovada sua efetividade contra o vírus SARS-CoV-2 conforme descrito pela ISO 18184:201913.

As concentrações de $\mathrm{Ag}$ nas amostras estudadas apresentaram uma grande variação entre elas de $14-72 \mu^{-1}{ }^{-1}$, indicando que diferentes tecnologias de incorporação da $\mathrm{Ag}$ na fibra têxtil foram utilizadas e podem ter levado a essa variação. E supondo que a Ag seja o componente virucida da máscara, a eficiência dessa ação pode ser diferente entre os produtos estudados. Mas nada pode ser afirmado, uma vez que não se tem estabelecido o

Tabela 1. Resultados dos ensaios de validação para prata (Ag) e nanopartícula de prata (AgNP) obtidos por espectrometria de massa com plasma indutivamente acoplado (ICP-MS).

\begin{tabular}{lcccc}
\hline Elemento & modo & LOD & LOQ & Faixa de trabalho \\
\hline \multirow{2}{*}{$\mathrm{Ag}$} & Padrão & $0,002 \mu \mathrm{gg}^{-1}$ & $0,05 \mu \mathrm{gg}^{-1}$ & - \\
& single particle & $\mathrm{LOD}_{\mathrm{d}}-16 \mathrm{~nm}$ & - & $1,5,10,15,20 \mu \mathrm{gL}^{-1}$ \\
\hline
\end{tabular}

Fonte: Elaborada pelos autores, 2020.

Ag: prata; LOD: limite de detecção; LOQ: limite de quantificação; LOD $_{d}$ : limite de detecção de tamanho; LOD $_{\mathrm{NP}}$ : limite de detecção da concentração do número de nanopartículas.

Tabela 2. Resultados da recuperação e da repetibilidade para prata total e nanopartículas de prata por espectrometria de massa com plasma indutivamente acoplado (ICP-MS).

\begin{tabular}{|c|c|c|c|c|c|c|}
\hline Amostra & Ag total adicionada $\mu \mathrm{gL}^{-1}$ & Ag total medida $\mu \mathrm{gL}^{-1}$ & AgNP adicionada partículas/mL & AgNP medida partículas/mL & \%REC & \%RSD \\
\hline A & $\begin{array}{l}30 \\
-\end{array}$ & $\begin{array}{c}31,6 \\
-\end{array}$ & $50 . \overline{-}$ & 39.737 & $\begin{array}{c}105 \\
83\end{array}$ & $\begin{array}{l}1,0 \\
2,5\end{array}$ \\
\hline B & $\begin{array}{c}30 \\
-\end{array}$ & $\begin{array}{c}22,4 \\
-\end{array}$ & 50.000 & 30.827 & $\begin{array}{l}75 \\
64\end{array}$ & $\begin{array}{l}10,0 \\
18,0\end{array}$ \\
\hline G & $\begin{array}{c}30 \\
-\end{array}$ & $\begin{array}{c}26,3 \\
-\end{array}$ & 50.000 & $50 . \overline{271}$ & $\begin{array}{c}92 \\
105\end{array}$ & $\begin{array}{l}7,0 \\
5,0\end{array}$ \\
\hline
\end{tabular}

Fonte: Elaborada pelos autores, 2020.

Ag: prata; AgNP: nanopartícula de prata; REC: recuperação; RSD: desvio-padrão relativo. 
Tabela 3. Resultados obtidos para prata total e para nanopartícula de prata nas máscaras analisadas neste estudo.

\begin{tabular}{|c|c|c|c|c|}
\hline \multirow{2}{*}{ Amostras } & \multirow{2}{*}{ Ag total $\mu g^{-1}$} & \multirow{2}{*}{ Tamanho da AgNP (nm) } & \multicolumn{2}{|c|}{ Concentração } \\
\hline & & & Partículas/g & Partículas $/ \mathrm{cm}^{2}$ \\
\hline A & $14,3 \pm 7,2$ & $24 \pm 3$ & $2,3 \times 10^{6} \pm 5,7 \times 10^{4}$ & $2,43 \times 10^{4} \pm 6,1 \times 10^{2}$ \\
\hline B & $25,9 \pm 4,1$ & $17 \pm 4$ & $<$ LOD & - \\
\hline C & $40,2 \pm 9,2$ & $57 \pm 3$ & $8,3 \times 10^{5} \pm 4,1 \times 10^{4}$ & $9,9 \times 10^{3} \pm 4,9 \times 10^{2}$ \\
\hline D & $72,0 \pm 4,1$ & $36 \pm 8$ & $<$ LOD & - \\
\hline $\mathrm{E}$ & $8,6 \pm 0,1$ & $19 \pm 3$ & $1,5 \times 10^{6} \pm 7,5 \times 10^{4}$ & $1,7 \times 10^{4} \pm 8,5 \times 10^{2}$ \\
\hline $\mathrm{F}$ & $0,3 \pm 0,1$ & - & - & - \\
\hline G & $0,5 \pm 0,1$ & - & - & - \\
\hline $\mathrm{H}$ & $1,9 \pm 0,4$ & - & - & - \\
\hline । & $0,6 \pm 0,1$ & - & - & - \\
\hline J & $0,4 \pm 0,1$ & - & - & - \\
\hline
\end{tabular}

Fonte: Elaborada pelos autores, 2020.

Ag: prata; AgNP: nanopartícula de prata; LOD: limite de detecção.

quanto de Ag deve ser aplicado para obtenção da eficácia antiviral. Outro fator observado foi a falta de homogeneidade na distribuição da Ag no tecido usado na confecção de cada amostra. Os resultados apresentaram RSD de 15\%-51\% indicando, assim, a heterogeneidade, o que pode afetar diretamente a ação da Ag no combate ao vírus SARS-CoV-2.

Além disso, $50 \%$ das amostras apresentaram Ag na forma de nanopartícula, coerentes com as informações declaradas nas embalagens. As outras amostras continham Ag na forma de íon e, segundo alguns autores, a Ag iônica possui baixa estabilidade ${ }^{8,9}$, o que levanta o questionamento quanto a sua real efetividade biocida ao longo do tempo. Porém, o íon Ag ainda é utilizado pelo seu baixo custo para o fabricante quando comparado com o valor das nanopartículas.

\section{Avaliação da concentração de Ag após ciclos de lavagem}

A variação da concentração de Ag nas amostras em relação a cada cinco ciclos de lavagem está apresentada na Figura 2. A porcentagem de perda é maior após o primeiro ciclo de lavagem, exceto na amostra $B$, cuja perda foi menor após o primeiro ciclo e que só começou a perder Ag após o quinto ciclo de lavagem (25 lavagens). As amostras A e E atingiram uma concentração abaixo de $1 \mu g^{-1}$ antes das 30 lavagens, o que contraria o declarado pelo fabricante, quanto à durabilidade da ação antiviral. Apesar de haver uma redução na concentração de Ag na amostra $C$ após as cinco primeiras lavagens, é possível observar que essa permaneceu em torno de $15 \mu \mathrm{gg}^{-1}$ ao longo dos ciclos de lavagem, podendo ser, possivelmente, justificada pela composição da fibra têxtil e da tecnologia usadas na confecção do produto. A amostra B apresentou maior estabilidade frente aos ciclos de lavagem, o que pode ser atribuído à moderna tecnologia aplicada no processo fabril. $A$ amostra $D$ não foi analisada, pois, infelizmente, não tínhamos quantidade suficiente e a amostra $F$, também, não foi analisada, uma vez que a concentração de prata inicial foi próxima as amostras que não apresentavam prata na sua composição.

Além da redução da ação biocida ao longo do processo de lavagem, uma questão preocupante que não pode ser negligenciada

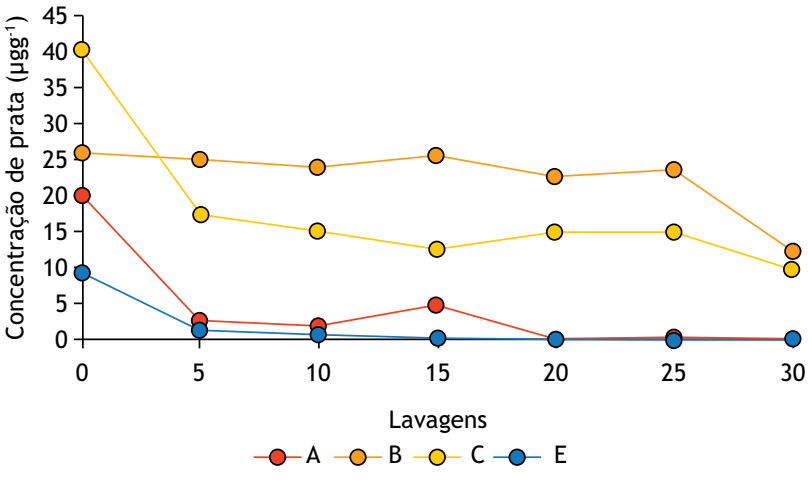

Fonte: Elaborada pelos autores, 2020.

Figura 2. Representação gráfica das concentrações de prata após os ciclos de lavagem das amostras A-E determinado por espectrometria de massa com plasma indutivamente acoplado (ICP-MS).

é a contaminação ambiental proveniente da água de lavagem dessas máscaras. Esse resíduo possui uma concentração considerável de Ag, seja sob a forma iônica ou nanopartículada, pois somente neste estudo, avaliando o processo de lavagem de apenas quatro amostras por seis ciclos, houve uma quantidade estimada de Ag descartada para o meio ambiente de $72,7 \mu \mathrm{gg}^{-1}$. Se projetarmos que cada indivíduo possua, em média, quatro máscaras de baixa vida útil (em torno de 30 lavagens), esse quantitativo de contaminação ambiental é maior, considerando o tamanho da população brasileira e o seu respectivo consumo de máscaras de proteção para a COVID-19.

\section{CONCLUSÕES}

O desenvolvimento e a implementação de novas metodologias que verifiquem a qualidade de novos produtos que surgem em função das emergências de saúde pública se tornaram grandes desafios científicos dos laboratórios fiscalizadores.

A técnica ICP-MS usada em laboratórios de rotina para diferentes produtos de interesse sanitário permite quantificar com precisão 
e exatidão a Ag seja na forma de íon, através do modo padrão, ou de AgNP através do modo single particle.

Este estudo promoveu o desenvolvimento e a validação de metodologia de análise de tecido, assim como a definição da melhor forma de preparo dessas amostras contendo AgNP. A partir dos resultados obtidos verificou-se a diversidade quanto à quantidade de $\mathrm{Ag}$, o que levanta um questionamento da eficácia dessas máscaras no combate à COVID19, uma vez que não se tem um valor mínimo preconizado da concentração de Ag para que se tenha eficácia de $99,9 \%$ contra o vírus.
As pesquisas na área de vigilância sanitária assumem um papel importante na resposta as emergências sanitárias, sobretudo aqueles aplicáveis em novos produtos que possam ser utilizados no combate as doenças e prevenir riscos futuros.

Sendo assim, há a necessidade de estabelecer os requisitos mínimos de composição e qualidade desses produtos por órgãos competentes de regulação e fiscalização, frente a atual oferta e demanda destes produtos contendo AgNP no mercado nacional. Estas carências legislativa e fiscalizatória acabam expondo a população a um elevado risco sanitário e podem ocasionar um problema de saúde pública emergente.

\section{REFERÊNCIAS}

1. Brito SBP, Isaque OB, Cunha CC, Palácio MGV, Takenami I. Pandemia da COVID-19: o maior desafio do século XXI. Vigil Sanit Debate. 2020;8(2):54-63. https://doi.org/10.22239/2317-269x.01531

2. British Broadcasting Corporation - BBC. Coronavírus: o mapa que mostra o alcance mundial da doença. BBC News. 2 mar 2020[acesso 20 de julho de 2020 ]. Disponível em: https: / / www.bbc.com/portuguese/internacional-51718755

3. Fundação Oswaldo Cruz - Ficruz. A utilização das máscaras é recomendada para evitar o coronavírus? COVID-19 Perguntas e Respostas. 27 maio 2020[acesso 16 de julho de 2020]. Disponível em: https://portal.fiocruz.br/pergunta/ utilizacao-das-mascaras-e-recomendada-para-evitar-ocoronavirus

4. World Health Organization - WHO. Advice on the use of masks in the context of COVID-19. Geneva: World Health Organization; 2020. Disponivel em https://www.who.int/ publications/i/item/advice-on-the-use-of-masks-in-thecommunity-during-home-care-and-in-healthcare-settings-inthe-context-of-the-novel-coronavirus-(2019-ncov)-outbreak

5. Azeredo HMC. Nanocomposites for food packaging applications. Food Res Intern. 2009;42(9):1240-53. https://doi.org/10.1016/j.foodres.2009.03.019

6. Barillo DJ, Marx DE. Silver in medicine: a brief history BC 335 to present. Burns. 2014;40(Supl.1):s3-s8. https://doi.org/10.1016/j.burns.2014.09.009

7. Miller G. Nano materials sunscreen and cosmetics: small materials big risks. Amsterdam: Friends of the Earth; 2006.

8. Tremiliosi G, Simoes LGP, Minozzi DT, Santos RI, Vilela DCB, Durigon EL et al. Ag nanoparticles-based antimicrobial polycotton fabrics to prevent the transmission and spread of SARS-CoV-2. BioRxiv. 2020:1-19. https://doi.org/10.1101/2020.06.26.152520

9. Rai M, Yadav A, Gad A. Silver nanoparticles as a new generation of antimicrobial. Biotech Adv. 2009;27(1):76-83. https://doi.org/10.1016/j.biotechadv.2008.09.002

10. International Organization for Standardization - ISO. Nanotechnologies, vocabulary, part 1: core terms. Geneva: International Organization for Standardization: 2010.

11. International Organization for Standardization - ISO. Nanotechnologies, vocabulary, part 2: nano-objects. Geneva: International Organization for Standardization: 2015

12. Gubala V, Johnston LJ, Liu Z, Harald K, Moore CJ, Ober CK et al. Engineered nanomaterials and human health: part 1: preparation, functionalization and characterization (IUPAC technical report). Pure Appl Chem. 2018;90(8):1283-324. https://doi.org/10.1515/pac-2017-0101

13. International Organization for Standardization - ISO. ISO 18184:2019. Textiles: determination of antiviral activity of textile products. Geneva: International Organization for Standardization: 2019

14. Santos LMG, Vicentini Neto as, lozzi G, Jacob SC. Arsenic, cadmium and lead concentrations in Yerba mate commercialized in Southern Brazil by inductively coupled plasma mass spectrometry. Cienc Rural. 2017;47(12):1-6 https://doi.org/10.1590/0103-8478cr20170202

15. Mackevica A, Olsson ME, Hansen SF. Quantitative characterization of $\mathrm{TiO}_{2}$ nanoparticle release from textiles by conventional and single particle ICP-MS. J Nanopart Res. 2018;20. https://doi.org/10.1007/s11051-017-4113-2

16. Agência Nacional de Vigilância Sanitária - Anvisa. Orientações gerais: máscaras faciais de uso não profissional. Brasília: Agência Nacional de Vigilância Sanitária; 2020[acesso 25 de julho de 2020]. Disponível em: http://portal.anvisa.gov.br/ documents/219201/4340788/NT+M\%C3\%A1scaras.pdf/ bf430184-8550-42cb-a975-1d5e1c5a10f7

17. Instituto Nacional de Metrologia, Normalização e Qualidade Industrial - Inmetro. Orientação sobre validação de métodos analíticos. Brasília: Instituto Nacional de Metrologia, Normalização e Qualidade Industrial; 2018.

18. International Organization for Standardization - ISO. Norma ABNT NBR ISO/IEC 17025:2017. Requisitos gerais para a competência de laboratórios de ensaio e calibração. Geneva: International Organization for Standardization; 2020.

19. Witzler M, Kullmer F, Hirtz A, Günther K. Validation of gold and silver nanoparticle analysis in fruit juice by single-particle ICP-MS without sample pretreatment. J Agric Food Chem. 2016;64(20):4165-70. https://doi.org/10.1021/acs.jafc.6b01248 
20. Laborda F, Jiménez-Lamana J, Bolea E, Castillo JR. Selective identification, characterization and determination of dissolved silver(i) and silver nanoparticles based on single particle detection by inductively coupled plasma mass spectrometry. J Anal At Spectrom. 2011;26(7):1362-71. https://doi.org/10.1039/C0JA00098A
21. Souza SV. Procedimento para validação intralaboratorial de métodos de ensaio: delineamento e aplicabilidade em análise de alimento [tese]. Belo Horizonte: Universidade Federal de Minas Gerais; 2007.

22. Bazilio FS, Bomfim MVJB, Almeida RJ, Abrantes SMP. Uso de planilha eletrônica na verificação da adequação de curva analítica ao modelo linear. Analytica. 2012;(59):60-7.

\section{Agradecimentos}

O presente trabalho foi realizado com o apoio da Coordenação de Aperfeiçoamento de Pessoal de Nível Superior-Brasil (CAPES) - Código de Financiamento 001, do Programa INOVA/Fiocruz - Edital Geração do conhecimento 2019, do projeto Edital FAPERJ n 02/2019 - Programa Redes de Pesquisa em Nanotecnologia no Estado do Rio de Janeiro.

\section{Contribuição dos Autores}

Barata-Silva C, Santos LMG - Concepção, planejamento (desenho do estudo), aquisição, análise, interpretação dos dados e redação do trabalho. Vicentini Neto SA, Magalhães CD - Aquisição, análise, interpretação dos dados e redação do trabalho. Jacob SC, Moreira JC Concepção, planejamento (desenho do estudo) e redação do trabalho. Todos os autores aprovaram a versão final do trabalho.

Conflito de Interesse

Os autores informam não haver qualquer potencial conflito de interesse com pares e instituições, políticos ou financeiros deste estudo.

Licença CC BY-NC atribuição não comercial. Com essa licença é permitido acessar, baixar (download), copiar, imprimir, compartilhar, reutilizar e distribuir os artigos, desde que para uso não comercial e com a citação da fonte, conferindo os devidos créditos de autoria e menção à Visa em Debate. Nesses casos, nenhuma permissão é necessária por parte dos autores ou dos editores. 